\title{
Retraction Note: Feasibility of Using Copper(II)Oxide for Additive Manufacturing
}

Yunho Yang, Christopher Yarka, Jian Cao, and Kornel Ehmann

( 2015 by KSPE and Springer

Retraction Note: Int. J. Precis. Eng. Manuf., Vol. 15, No. 9,

pp. 1961-1965, 2014

DOI 10.1007/s12541-014-0551-2

The authors have requested this publication be retracted because sensitive ideas, samples and methods used for and described within this publication, developed, produced, and owned by others, were inadvertently included. 